International Mathematical Forum, Vol. 9, 2014, no. 15, 725 - 731

HIKARI Ltd, www.m-hikari.com

http://dx.doi.org/10.12988/imf.2014.4344

\title{
Convergence Theorems for Pettis Integral of Functions Taking Values in Locally Convex Spaces
}

\author{
Zamir Selko \\ Faculty of Natural Sciences \\ Department of Mathematics \\ University of Elbasan, Albania
}

Copyright (C) 2014 Zamir Selko. This is an open access article distributed under the Creative Commons Attribution License, which permits unrestricted use, distribution, and reproduction in any medium, provided the original work is properly cited.

\begin{abstract}
In this paper we present convergence theorems for Pettis integral of functions defined on a complete probability space and taking values in a complete locally convex topological vector space.
\end{abstract}

Mathematics Subject Classification: 28B05, 46G10

Keywords: Pettis integral, convergence theorems, complete locally convex topological vector space

\section{Introduction}

We present convergence theorems for Pettis integral of functions defined on a complete probability space and taking values in a complete locally convex topological vector space, Theorems 2.2 and 2.3 , which are the analogues of the known convergence theorems for Pettis integral of functions taking values in a Banach space. For that purpose, we apply a technique which is based on the limit projective of Banach spaces, Lemma 2.1.

Theorem 2.2 is the analogue of Vitali Theorem for Pettis integral of functions taking values in a Banach space, Theorem 8.1 in [6]. Theorem 2.2 has been proved by another approach in [7]. Theorem 2.3 is the analogue of Theorem 2.8 in [9]. For more information on convergence theorems for Pettis integral, see [6], [7], [8], [4] and [2]. 
Throughout this paper $(\Omega, \Sigma, \mu)$ is a complete probability space and $V$ is a complete locally convex space with its topology $\tau$ and topological dual $V^{\prime}$. By $\mathrm{P}$ we denote the family of all continuous semi-norms in this space; for every $p \in \mathrm{P}, \widetilde{V}^{p}$ denotes the quotient vector space of the vector space $V$ with respect to the equivalence relation $x \sim_{p} y \Leftrightarrow p(x-y)=0$; the map $\phi_{p}: V \rightarrow \widetilde{V}^{p}$ is the canonical quotient map, thus is the equivalence class of an element with respect to the relation " $\sim_{p}$ "; the quotient normed space $\left(\widetilde{V}^{p}, \widetilde{p}\right)$ is called the normed component of the space $V$, where $\widetilde{p}\left(\phi_{p}(x)\right)=p(x)$, for each $x \in V$; the Banach space $\left(\bar{V}^{p}, \bar{p}\right)$ which is the completion of the space $\left(\widetilde{V}^{p}, \widetilde{p}\right)$ is called the Banach component of the space $V ; \widetilde{V}_{p}^{\prime}$ and $\bar{V}_{p}^{\prime}$ are the topological duals of $\left(\widetilde{V}^{p}, \widetilde{p}\right)$ and $\left(\bar{V}^{p}, \bar{p}\right)$ respectively. It is easy to see that

$$
V^{\prime}=\left\{\widetilde{v}_{p}^{\prime} \circ \phi_{p} / \widetilde{v}_{p}^{\prime} \in \widetilde{V}_{p}^{\prime}, p \in \mathrm{P}\right\}
$$

because for every $v^{\prime} \in V^{\prime}$, we have that $\left|v^{\prime}().\right| \in \mathrm{P}$. For every $p, q \in \mathrm{P}$ such that $p \leq q$, we define the map $\widetilde{g}_{p q}: \widetilde{V}^{q} \rightarrow \widetilde{V}^{p}$ by $\widetilde{g}_{p q}\left(w_{q}\right)=w_{p}$, for all $w_{q} \in \widetilde{V}^{q}$, where $w_{p}=\phi_{p}(x)$, for some vector $x \in w_{q}$. We define also the map $\bar{g}_{p q}: \bar{V}^{q} \rightarrow \bar{V}^{p}$ as the continuous linear extension of $\widetilde{g}_{p q}$, for every $p, q \in \mathrm{P}$ such that $p \leq q$.

The following definition is given by [1], Definition 1 .

Definition 1.1 A function $f: S \rightarrow V$ is called Pettis integrable if the function $v^{\prime} \circ f$ is Lebesgue integrable for each $v^{\prime} \in V^{\prime}$ and if for every measurable set $E$ of $\Omega$, there is a vector $x_{E} \in V$ such that $v^{\prime}\left(x_{E}\right)=\int v^{\prime}(f(s))$ for every $v^{\prime} \in V^{\prime}$. The vector $x_{E}$ is said to be Pettis integral of function $f$ on $E$ and we denote:

$$
x_{E}=(P) \int_{E} f
$$

If $V$ is a Banach space, this definition is the same with Definition 2, [3], p.52.

Definition 1.2 A family $H$ of real-valued integrable functions defined on $\Omega$ is said to be uniformly integrable if it satisfies the conditions

1. $\sup _{h \in H} \int_{\Omega}|h|<\infty$,

2. For each $\epsilon>0$ there is $\delta(\epsilon)>0$ such that the inequality

$$
\sup _{h \in H} \int_{E}|h|<\epsilon
$$

holds for every $E \in \Sigma$ satisfying $\mu(E) \leq \delta(\epsilon)$. 
Let $f: \Omega \rightarrow V$ be a function at let $p \in \mathrm{P}$. We put

$$
Z_{f}^{p}=\left\{\widetilde{v}_{p}^{\prime} \circ\left(\phi_{p} \circ f\right) / \widetilde{v}_{p}^{\prime} \in B\left(\widetilde{V}_{p}^{\prime}\right)\right\}
$$

where $B\left(\widetilde{V}_{p}^{\prime}\right)$ is the closed unit ball in $\widetilde{V}_{p}^{\prime}$. It is clear to see that

$$
Z_{f}^{p}=\left\{\bar{v}_{p}^{\prime} \circ\left(\phi_{p} \circ f\right) / \bar{v}_{p}^{\prime} \in B\left(\bar{V}_{p}^{\prime}\right)\right\}
$$

where $B\left(\bar{V}_{p}^{\prime}\right)$ is the closed unit ball in $\bar{V}_{p}^{\prime}$.

\section{Convergence theorems for Pettis integral}

The proof of the following auxiliary lemma is similar in spirit to the proof of Lemma 4.1 in [5].

Lemma 2.1 Let $f: \Omega \rightarrow V$ be a function. The function $f$ is Pettis integrable if and only if for every $p \in \mathrm{P}$ the function $\phi_{p} \circ f$ is Pettis integrable in the Banach component $\left(\bar{V}^{p}, \bar{p}\right)$. In this case, we have

$$
\phi_{p}\left((P) \int_{E} f\right)=(P) \int_{E} \phi_{p} \circ f \quad \text { for all } \quad p \in \mathrm{P}, E \in \Sigma \text {. }
$$

Proof. Suppose that the function $f$ is Pettis integrable and let $p \in \mathrm{P}$. Then, by Definition 1.1 and (1), the function $\widetilde{v}_{p}^{\prime} \circ\left(\phi_{p} \circ f\right)=v^{\prime} \circ f$ is the Lebesgue integrable, for each $\widetilde{v}_{p}^{\prime} \in B\left(\widetilde{V}_{p}^{\prime}\right)$. We have also that for each $E \in \Sigma$, there is a vector $x_{E} \in V$ such that $v^{\prime}\left(x_{E}\right)=\int_{E} v^{\prime} \circ f$, for all $v^{\prime} \in V^{\prime}$. Hence, we obtain by (1) that

$$
\left(\widetilde{v}_{p}^{\prime} \circ \phi_{p}\right)\left(x_{E}\right)=\int_{E}\left(\widetilde{v}_{p}^{\prime} \circ \phi_{p}\right) \circ f \quad \text { for each } \quad \widetilde{v}_{p}^{\prime} \in B\left(\widetilde{V}_{p}^{\prime}\right) .
$$

Therefore, the function $\phi_{p} \circ f$ is Pettis integrable in the Banach component $\left(\bar{V}^{p}, \bar{p}\right)$ and $\phi_{p}\left(x_{E}\right)=(P) \int_{E} \phi_{p} \circ f$, for all $E \in \Sigma$.

Conversely, assume that for every $p \in \mathrm{P}$ the function $\phi_{p} \circ f$ is Pettis integrable in the Banach component $\left(\bar{V}^{p}, \bar{p}\right)$, and let $v^{\prime} \in V^{\prime}$ and $E \in \Sigma$ are given. Then, by the equality (1), there exist $p \in \mathrm{P}$ and $\widetilde{v}_{p}^{\prime} \in B\left(\widetilde{V}_{p}^{\prime}\right)$ such that $v^{\prime}=\widetilde{v}_{p}^{\prime} \circ \phi_{p}$ and the function $v^{\prime} \circ f=\widetilde{v}_{p}^{\prime} \circ\left(\phi_{p} \circ f\right)$ is Lebesgue integrable. We have also that for each $p \in \mathrm{P}$ there exists $\bar{I}_{p}(E) \in \bar{V}^{p}$ such that

$$
\bar{v}_{p}^{\prime}\left(\bar{I}_{p}(E)\right)=\int_{E} \bar{v}_{p}^{\prime} \circ\left(\phi_{p} \circ f\right)=\int_{E} \widetilde{v}_{p}^{\prime} \circ\left(\phi_{p} \circ f\right) \text { for all } \bar{v}_{p}^{\prime} \in \bar{V}_{p}^{\prime}
$$

where $\widetilde{v}_{p}^{\prime}=\left.\bar{v}_{p}^{\prime}\right|_{\widetilde{V}^{p}}$. 
Assume that two arbitrary continuous seminorms $p$ and $q$ such that $p \leq$ $q$ are given. Since $\bar{I}_{q}(E) \in \bar{V}^{q}$, there is a sequence $\left(w_{q}^{n}\right) \subset \widetilde{V}^{q}$ such that $\lim _{n \rightarrow \infty} w_{q}^{n}=\bar{E}_{q}(E)$. Then, $\lim _{n \rightarrow \infty} w_{p}^{n}=\bar{g}_{p q}\left(\bar{I}_{q}(E)\right)$, where $w_{p}^{n}=\widetilde{g}_{p q}\left(w_{q}^{n}\right)$, for all $n \in \mathbb{N}$. Hence

$$
\lim _{n \rightarrow \infty} \widetilde{v}_{p}^{\prime}\left(w_{p}^{n}\right)=\bar{v}_{p}^{\prime}\left(\bar{g}_{p q}\left(\bar{I}_{q}(E)\right)\right) \quad \text { for all } \quad \bar{v}_{p}^{\prime} \in \bar{V}_{p}^{\prime},
$$

where $\widetilde{v}_{p}^{\prime}=\left.\bar{v}_{p}^{\prime}\right|_{\widetilde{V}^{p}}$. By virtue of (3), we have

$$
\begin{aligned}
\lim _{n \rightarrow \infty} \widetilde{v}_{p}^{\prime}\left(w_{p}^{n}\right) & =\lim _{n \rightarrow \infty}\left(\widetilde{v}_{p}^{\prime} \circ \widetilde{g}_{p q}\right)\left(w_{q}^{n}\right)=\int_{E}\left(\widetilde{v}_{p}^{\prime} \circ \widetilde{g}_{p q}\right) \circ\left(\phi_{q} \circ f\right) \\
& =\int_{E} \widetilde{v}_{p}^{\prime} \circ\left(\widetilde{g}_{p q} \circ \phi_{q}\right) \circ f=\int_{E} \widetilde{v}_{p}^{\prime} \circ\left(\phi_{p} \circ f\right)=\bar{v}_{p}^{\prime}\left(\bar{I}_{p}(E)\right)
\end{aligned}
$$

for all $\bar{v}_{p}^{\prime} \in \bar{V}_{p}^{\prime}$, where $\widetilde{v}_{p}^{\prime}=\left.\bar{v}_{p}^{\prime}\right|_{\widetilde{V}^{p}}$. The last result together with (4) yields

$$
\bar{v}_{p}^{\prime}\left(\bar{g}_{p q}\left(\bar{I}_{q}(E)\right)\right)=\bar{v}_{p}^{\prime}\left(\bar{I}_{p}\right) \text { for all } \quad \bar{v}_{p}^{\prime} \in \bar{V}_{p}^{\prime} .
$$

Therefore by Corollary IV.6.2 in [11], we obtain $\bar{g}_{p q}\left(\bar{I}_{q}(E)\right)=\bar{I}_{p}(E)$. Hence, by Theorem II.5.4 in [10], there is a vector $I_{f}(E) \in V$ such that

$$
\phi_{p}\left(I_{f}(E)\right)=\bar{I}_{p}(E) \quad \text { for each } \quad p \in \mathrm{P} .
$$

Thus, since $v^{\prime}=\widetilde{v}_{p}^{\prime} \circ \phi_{p}$, we have

$$
v^{\prime}\left(I_{f}(E)\right)=\widetilde{v}_{p}^{\prime}\left(\phi_{p}\left(I_{f}(E)\right)\right)=\widetilde{v}_{p}^{\prime}\left(\bar{I}_{p}(E)\right)=\int_{E} \widetilde{v}_{p}^{\prime} \circ\left(\phi_{p} \circ f\right)=\int_{E}\left(\widetilde{v}_{p}^{\prime} \circ \phi_{p}\right) \circ f .
$$

Hence

$$
v^{\prime}\left(I_{f}(E)\right)=\int_{E} v^{\prime} \circ f
$$

and since $v^{\prime}$ and $E$ are arbitrary, the last equality holds for each $v^{\prime} \in V^{\prime}$ and $E \in \Sigma$. This means that $f$ is Pettis integrable and $I_{f}(E)=(P) \int_{E} f$, for all $E \in \Sigma$. Hence, the equality (2) holds and the proof is finished.

Theorem 2.2 Let $\left(f_{n}\right)$ be a sequence of Pettis integrable functions $f_{n}: \Omega \rightarrow$ $V$ and let $f: \Omega \rightarrow V$ be a function such that

(i) for every $v^{\prime} \in V^{\prime}$, we have $v^{\prime} \circ f_{n} \rightarrow v^{\prime} \circ f \mu$-a.e.;

(ii) for every $p \in \mathrm{P}$, we have $\bigcup_{n \in \mathbb{N}} Z_{f_{n}}^{p}$ is uniformly integrable.

Then, $f$ is Pettis integrable and, for each $E \in \Omega$, we have

$$
\lim _{n \rightarrow \infty}(P) \int_{E} f_{n}=(P) \int_{E} f
$$

in the weak topology $\sigma\left(V, V^{\prime}\right)$. 
Proof. By Lemma 2.1, each function $\phi_{p} \circ f_{n}$ is Pettis integrable in the Banach component $\left(\bar{V}^{p}, \bar{p}\right)$ and

$$
(P) \int_{E} \phi_{p} \circ f_{n}=\phi_{p}\left((P) \int_{E} f_{n}\right) \quad \text { for each } E \in \Omega \text {. }
$$

By hypothesis and (1), for each $\widetilde{v}_{p}^{\prime} \in \widetilde{V}_{p}^{\prime}$, we have $\widetilde{v}_{p}^{\prime} \circ\left(\phi_{p} \circ f_{n}\right) \rightarrow \widetilde{v}_{p}^{\prime} \circ\left(\phi_{p} \circ f\right)$, $\mu$ - a.e. Thus, the conditions of Theorem 8.1 in [6] are satisfied. Therefore, the function $\phi_{p} \circ f$ is Pettis integrable in the Banach component $\left(\bar{V}^{p}, \bar{p}\right)$ and

$$
\lim _{n \rightarrow \infty} \bar{v}_{p}^{\prime}\left((P) \int_{E} \phi_{p} \circ f_{n}\right)=\bar{v}_{p}^{\prime}\left((P) \int_{E} \phi_{p} \circ f\right) \quad \text { for each } \quad E \in \Omega, \bar{v}_{p}^{\prime} \in \bar{V}_{p}^{\prime} .
$$

Hence, by Lemma 2.1, we obtain that $f$ is Pettis integrable and

$$
\lim _{n \rightarrow \infty}\left(\widetilde{v}_{p}^{\prime} \circ \phi_{p}\right)\left((P) \int_{E} f_{n}\right)=\left(\widetilde{v}_{p}^{\prime} \circ \phi_{p}\right)\left((P) \int_{E} f\right) \quad \text { for all } E \in \Omega, \widetilde{v}_{p}^{\prime} \in \widetilde{V}_{p}^{\prime},
$$

where $\widetilde{v}_{p}^{\prime}=\left.\bar{v}_{p}^{\prime}\right|_{\widetilde{V}^{p}}$. Further, we obtain by (1) that

$$
\lim _{n \rightarrow \infty} v^{\prime}\left((P) \int_{E} f_{n}\right)=v^{\prime}\left((P) \int_{E} f\right) \quad \text { for all } \quad E \in \Omega, v^{\prime} \in V^{\prime}
$$

and the proof is finished.

Theorem 2.3 Let $\left(f_{n}\right)$ be a sequence of Pettis integrable functions $f_{n}: \Omega \rightarrow$ $V$ converging point-wise to a function $f: \Omega \rightarrow V$ in $(V, \tau)$. Then the following are equivalent.

(i) for every $p \in \mathrm{P}$, the family $\bigcup_{n \in N} Z_{f_{n}}^{p}$ is uniformly integrable;

(ii) the function $f$ is Pettis integrable and, for each $E \in \Omega$, we have

$$
\lim _{n \rightarrow \infty}(P) \int_{E}\left(f_{n}\right)=(P) \int_{E} f \quad \text { in }(V, \tau)
$$

Proof. Note that the sequence $\left(f_{n}\right)$ converges point-wise to $f$ in $(V, \tau)$, if and only if for every $p \in \mathrm{P}$ the sequence $\phi_{p} \circ f_{n}$ converges point-wise to $\phi_{p} \circ f$ in the normed component $\left(\widetilde{V}^{p}, \widetilde{p}\right)$.

$(i) \Rightarrow\left(\right.$ ii) Let $p$ be an element of $\mathrm{P}$. We have that the sequence $\phi_{p} \circ f_{n}$ converges point-wise to $\phi_{p} \circ f$ in the Banach component $\left(\bar{V}^{p}, \bar{p}\right)$. we have also by Lemma 2.1 that each function $\phi_{p} \circ f_{n}$ is Pettis integrable in the Banach component $\left(\bar{V}^{p}, \bar{p}\right)$ and

$$
(P) \int_{E}\left(\phi_{p} \circ f_{n}\right)=\phi_{p}\left((P) \int_{E} f_{n}\right) \in \tilde{V}^{p} \quad \text { for all } \quad E \in \Omega .
$$


So, the conditions of Theorem 2.8 in [9] are satisfied. Therefore, the function $\phi_{p} \circ f$ is Pettis integrable in the Banach component $\left(\bar{V}^{p}, \bar{p}\right)$ and the equality

$$
\lim _{n \rightarrow \infty}(P) \int_{E} \phi_{p} \circ f_{n}=(P) \int_{E} \phi_{p} \circ f,
$$

holds in $\left(\bar{V}^{p}, \bar{p}\right)$, for each $E \in \Omega$.

Let $E$ be an element of $\Sigma$. We have that for every $p \in \mathrm{P}$, the function $\phi_{p} \circ f$ is Pettis integrable in the Banach component $\left(\bar{V}^{p}, \bar{p}\right)$. Therefore, by Lemma 2.1, the function $f$ is Pettis integrable and

$$
(P) \int_{E} \phi_{p} \circ f=\phi_{p}\left((P) \int_{E} f\right) \in \widetilde{V}^{p}
$$

Hence, inserting the right-hand-sides of (5) and (7) to (6), we obtain

$$
\lim _{n \rightarrow \infty} \phi_{p}\left((P) \int_{E} f_{n}\right)=\phi_{p}\left((P) \int_{E} f\right) \quad \text { for each } \quad p \in \mathrm{P} .
$$

Therefore, $\lim _{n \rightarrow \infty}(P) \int_{E} f_{n}=(P) \int_{E} f$ in $(V, \tau)$.

$(i i) \Rightarrow(i)$ Assume that $(i i)$ holds and let $E \in \Sigma$ and $p \in \mathrm{P}$. We have that the equality

$$
\lim _{n \rightarrow \infty} \phi_{p}\left((P) \int_{E} f_{n}\right)=\phi_{p}\left((P) \int_{E} f\right),
$$

holds in the Banach space $\left(\bar{V}^{p}, \bar{p}\right)$. According to Lemma 2.1, the functions $\phi_{p} \circ f$ and $\phi_{p} \circ f_{n}$ are Pettis integrable in $\left(\bar{V}^{p}, \bar{p}\right)$ and

$$
\phi_{p}\left((P) \int_{E} f\right)=(P) \int_{E} \phi_{p} \circ f \quad \phi_{p}\left((P) \int_{E} f_{n}\right)=(P) \int_{E} \phi_{p} \circ f_{n} .
$$

Hence, inserting the right-hand-sides of these equalities to (8), we obtain that the equality

$$
\lim _{n \rightarrow \infty}(P) \int_{E} \phi_{p} \circ f_{n}=(P) \int_{E} \phi_{p} \circ f
$$

holds in $\left(\bar{V}^{p}, \bar{p}\right)$. Therefore, by Theorem 2.8 in [9], the family $\bigcup_{n \in N} Z_{f_{n}}^{p}$ is uniformly integrable and the proof is finished.

Acknowledgments. Author thanks Professor Sokol Kaliaj for the proof of Lemma 2.1.

\section{References}

[1] N. D. Chakraborty and Sk. Jaker Ali, On strongly Pettis integrable functions in locally convex spaces, Revista Mathematica Univ.Comp. Madrid Vol.6, no.2, (1993). 
[2] N. D. Chakraborty and T. Choudhury, Convergence theorems for Pettis integrable functions and regular methods of summability, Math.Anal.Appl. 359 (2009), 95-105.

[3] J. Distel and J. J. Uhl, Vector Measures, American Mathematical Society, Providence, (1977).

[4] R. F. Geitz, Pettis integration, Proc.Amer.Math.Soc. no.1, 82(1981), 8187.

[5] S. B. Kaliaj, A. D. Tato, F. D. Gumeni, Controlled convergence theorems for Henstock-Kurzweil-Pettis integral on m-dimensional compact intervals, Czechoslovak Math. J. 62 (137) (2012), 243-255.

[6] K. Musial, Topics in the theory of Pettis integration, Rend. Ist. Mat. Univ. Trieste 23,(1993),177-262.

[7] K. Musial, Vitali and Lebesgue convergence theorems for Pettis integral in locally convex spaces, Atti Sem. Mat. Fis. Univ. Modena, 35(1987),159166).

[8] K. Musial, Pettis Integral, Handbook of measure theory, Vol.I,II, NorthHolland, Amsterdam,(2002), 531-586.

[9] J. Rodriguez, Pointwise limits of Birkhoff integrable functions, Proc. Amer. Math. Soc. no.1,137 (2009), 235-245.

[10] H. H. Schaefer, Topological Vector Spaces, Springer-Verlag New York (1971).

[11] K. Yosida, Functional Analysis, Springer-Verlag Berlin Heidelberg (1980).

\section{Received: January 12, 2014}

